\title{
El emprendimiento en la Educación Superior en la Titulación de Turismo, ¿cuestión de género?
}

\author{
Guzmán A. Muñoz Fernández, \\ Pablo Rodríguez Gutiérrez y Luna Santos Roldán
}

Departamento de Estadística, Econometría, Investigación Operativa, Organización de Empresas y Economía Aplicada, Universidad de Córdoba

\section{España}

Correspondencia: Guzmán Antonio Muñoz Fernández, Facultad de Derecho y CC. Económicas y Empresariales, Pl/ Puerta Nueva, s/n (14004) Córdoba (España). E-mail:guzman.munoz@uco.es

(C) Education \& Psychology I+D+i and Ilustre Colegio Oficial de la Psicología de Andalucía Oriental (Spain) 


\section{Resumen}

Introducción. Este trabajo estudia las competencias intrapersonales que determinan el perfil emprendedor del alumnado en la Educación Superior en Turismo para comprobar si existen diferencias significativas según género como diagnóstico y punto de partida de la situación para futuros planes de estudio.

Método. Se realizó una encuesta a estudiantes de Educación Superior en Turismo de la Universidad de Córdoba (España) y se analizaron las competencias intrapersonales relacionadas con el emprendimiento y ciertas variables moderadoras sociodemográficas y de formación. Para el análisis de los datos se han realizado modelos de regresión logit sobre intención emprendedora, por género.

Resultados. Los resultados muestran que las mujeres tienen mayor disciplina y responsabilidad que los hombres, características básicas para la creación de empresas, sin embargo, presenta una menor propensión hacia el emprendimiento, y ninguna competencia significativa relacionada con el riesgo o la iniciativa.

Discusión y Conclusión. Se intuye que las estrategias de educación desde la niñez pueden ser desalentadoras de este tipo de actitudes. Se propone la necesidad de realizar políticas educativas específicas para las alumnas en los distintos niveles educativos, que fomenten estas competencias relacionadas con el emprendimiento.

Palabras Clave: Emprendimiento, Género, Competencias, Educación Superior, Turismo 


\title{
Entrepreneurship in Higher Education in Tourism, gender issue?
}

\begin{abstract}
Introduction. This paper analyzes those interpersonal skills which determine the entrepreneurial profile among students in Tourism Higher Education. We aim to verify if there are significant differences by gender diagnosis and to take this into account for future academic curriculums.
\end{abstract}

Method. A survey was conducted with the students of Tourism at the University of Cordoba (Spain); we have studied the interpersonal skills related to entrepreneurship and several sociodemographic and education moderating variables. For data analysis, we have developed logit regression models about entrepreneurial intention by gender.

Results. The results show that women have more discipline and responsibility than men, hich is fundamental for start-ups; but in opposition to this, women have a lower propensity towards entrepreneurship and it has not been perceived significant competences connected to the risk or initiative.

Conclusion. It is deduced from the paper that some educational strategies since childhood could be discouraging this kind of attitude. We outline the need for developing specific education policies for female students at different educational levels, in order to promote the skills linked to entrepreneurship.

Keywords: Entrepreneurship, Gender, Skills, Higher Education, Tourism 


\section{Introducción}

La iniciativa emprendedora es entendida como la aptitud de los individuos para transformar las ideas en actos. Es una competencia clave en los jóvenes que los impulsa a ser más creativos y les ayuda a tener más confianza en sí mismos, por lo que debe estar presente en todo momento de su proceso formativo. El espíritu empresarial contribuye a la economía de un país mediante la innovación, generando competencia, creando empleo y contribuyendo a la creación de riqueza económica e incrementando su poder adquisitivo (Holmgren \& From, 2005).

Cuando este espíritu emprendedor se centra en el sector del turismo se concreta en nuevas experiencias en los turistas e incrementan su satisfacción (Blake, Sinclair \& Soria, 2006; Cawley \& Gillmor, 2008). No se debe olvidar que el sector turístico está inmerso en un profundo cambio estructural cuyos efectos se intensificarán en el futuro (Danilo, Melo \& Rocha, 2012; Pedreño \& Ramón, 2009), marcando innovaciones y por procesos totalmente nuevos que sustituirán a los antiguos (Tether, 2003), con una creciente competitividad y complejidad que hace imprescindible que la formación superior en turismo deba adquirir habilidades relacionadas con el emprendimiento y la búsqueda de oportunidades. Sin embargo, la propensión de los estudiantes universitarios a escoger la auto-ocupación como salida profesional es todavía baja, en comparación con otras alternativas (Benavides, SánchezGarcía \& Luna-Arocas, 2004) y se sigue confiando en la búsqueda de un empleo, cada vez más precario.

A la pregunta de si es necesario tener capacidades o cualidades especiales para ser emprendedor, algunos autores consideran que aquellos que saben identificar las oportunidades, son capaces de reunir y motivar los recursos necesarios para lograr los objetivos, y asimismo, son igualmente capaces de liderar un equipo, que a su vez liderará la organización (Silva, 1998). Por otro lado, esta mutación del sector turístico hace que se formen profesionales que trabajarán para un turismo que todavía no existe, por lo que hay que proporcionar a los estudiantes un aprendizaje más versátil, más voluble y más dúctil (Espelt, 2009). Es decir, se deben adquirir competencias en la toma de decisiones, adaptabilidad, creatividad, flexibilidad y nuevas tecnologías, porque el emprendedor también se hace. Sin embargo, la mayoría de planes de estudio están destinados a una capacitación que, en ocasiones, no va más allá de la recepción de un hotel o en una agencia de viajes. 
Con este trabajo hemos querido conocer las competencias intrapersonales que determinan el perfil emprendedor de los alumnos en la educación superior de turismo en España y comprobar si existen diferencias significativas según género, con la finalidad de servir como diagnóstico y punto de partida, y determinar qué competencias deben adquirir en sus planes de estudio. Conforme lo referido, para conseguir nuestro objetivo, el trabajo se divide en cinco partes: partiendo de esta introducción al tema, se continúa con una revisión de la bibliografía, para seguir con la presentación de la metodología utilizada en la investigación y los resultados de campo obtenidos. A modo de cierre, se exponen las conclusiones a nuestra investigación y las referencias bibliográficas.

\section{Revisión de la literatura}

La literatura ha planteado el estudio del emprendimiento desde tres perspectivas: sociodemográfica, psicológica y situacional, abordándose desde diferentes áreas de las Ciencias Sociales. La perspectiva sociodemográfica, en referencia a la profesión o experiencia empresarial de la familia, edad, sexo, etc., son factores que han sido vinculados con la propensión de emprendimiento. La tradición empresarial familiar influye en la actitud hacia el emprendimiento (Basu \& Goswami, 1999; Duchesneau \& Gartner, 1990). A través del trabajo en la empresa familiar, las personas adquieren ideas de negocio y habilidades para iniciar el suyo propio, o continuar el de su familia, muy especialmente la industria del turismo tradicionalmente dominada por empresas familiares (Getz \& Carlsen, 2005). También se señala el factor regional y el espíritu empresarial étnico, en particular, entre los determinantes hacia el emprendimiento (Tolbert, David \& Sine, 2011). De forma paralela, existen estudios que aluden a la influencia de la experiencia internacional en la situación emprendedora internacional (Sommer, 2013). Investigaciones empíricas también ponen de manifiesto la relación entre emprendimiento y género (Díaz \& Jiménez, 2004; González-Morales, 2001; Mueller, 2004; Veciana \& Urbano, 2004;), donde insinúan una mayor tendencia al emprendimiento en los hombres que las mujeres. Delmar \& Davidsson (2000) afirman que el género es determinante en la decisión de emprender, ya que los hombres tienen mayor intención de crear una empresa que las mujeres. Desde análogas consideraciones, también el entorno influye en la percepción de deseabilidad y posibilidad en la creación de nuevas empresas (Díaz, Hernández \& Raposo, 2007).

La perspectiva psicológica busca los rasgos característicos intrínsecos que diferencian al emprendedor frente al no emprendedor. Esta perspectiva se basa en la creatividad (Elkington \& Hartigan, 2008), el optimismo, pesimismo y realismo (López \& García, 2011), la con- 
fianza en sí mismo, la propensión a afrontar riesgos y la tolerancia a la incertidumbre (Begley \& Boyd, 1987; Thomas \& Mueller, 2000) e incluso a la autonomía personal o necesidad de apoyo del individuo. Algunos estudios consideran que la influencia de ciertos rasgos de personalidad es el mayor determinante en las intenciones de emprendimiento (Nga \& Shamuganathan, 2010), siendo una de las explicaciones para justificar estos resultados, que los emprendedores puedan percibir el contexto del riesgo de forma diferente que otros segmentos de la población (Caliendo \& Kritikos, 2009; Janney \& Dess, 2006). Las investigaciones realizadas por Ang \& Hong (2000), Gürol \& Atsan (2006) y Koh (1996) en estudiantes, mostraron que la intención emprendedora es mayor en aquellos que tienen más propensión a tomar riesgos, existiendo una relación positiva entre la propensión a asumir riesgos y la intención de crear una empresa (de la Fuente, Vera \& Cardelle-Elawar, 2012; Gurel, Altinay \& Daniele, 2010).

La perspectiva situacional hace que el individuo se plantee el emprendimiento cuando su situación económica o social cambia, o vea una oportunidad en su ejercicio profesional. Desde este punto de vista, una situación de desempleo es considerada como un factor determinante (Evans \& Leighton, 1989). Es necesario destacar que la situación de desempleo de larga duración o la escasa confianza en obtener un trabajo hace convertirse en empresarios por necesidad para evitar, en muchos casos, la marginalidad. Para Shapero \& Sokol (1982), el hombre se mueve por una inercia que varía cuando su situación se interrumpe o cambia de forma positiva o negativa, este hecho precipita tomar una decisión para buscar una mejor oportunidad. En este sentido, el desarrollo económico de la sociedad y la actuación de las administraciones están asumiendo un papel activo en la cultura del emprendimiento que influye en este proceso emprendedor (Alburquerque, 2008; Castro, Benerrechea \& Ibarra, 2011; Meek, Pacheco \& York, 2010; Tolbert et al., 2011). A partir de estas aportaciones se ha estudiado la importancia que las instituciones tienen en fomentar la capacidad de emprendimiento de las mujeres (Bruton, Ahlstrom \& Li, 2010; De Bruin, Brush \& Welter, 2007).

\section{Objetivos}

Según lo expuesto, se desvela la necesidad de formar para el emprendimiento en el proceso formativo de los alumnos, e incluir la competencia para crear, reconocer oportunidades y evaluar los riesgos (Detienne \& Chandler, 2004). Cuando más se fomente en los estudios de educación superior estas habilidades, será más probable que los alumnos puedan desarrollar este espíritu emprendedor en un momento de su vida, cuando el factor social o 
situacional fuera adecuado. En este sentido, para alcanzar nuestro objetivo principal de conocer si las competencias intrapersonales y /o el contexto sociocultural se encuentran relacionados con la intención de iniciar una actividad empresarial propia, observado desde la perspectiva de género; y en caso afirmativo, cuantificar dicha relación, se formulan las siguientes preguntas de investigación que serán verificadas en el estudio, como sigue:

- Pregunta de investigación 1 (RQ1): ¿existe relación entre el género y la deseabilidad de emprender?

- Pregunta de investigación 2 (RQ2): ¿existe relación entre las competencias intrapersonales y la deseabilidad de emprender?

- Pregunta de investigación 3 (RQ3): ¿en qué medida el género determina el perfil personal hacia el emprendimiento?

\section{Método}

\section{Participantes}

El cuestionario fue pasado inicialmente a 353 alumnos de Turismo de la Universidad de Córdoba, durante los meses de abril y mayo del 2014, se eliminaron 6 encuestas por omisión de datos significativos. La selección de los encuestados se realizó mediante un muestreo aleatorio simple. La muestra es un fiel reflejo de la realidad demográfica del alumnado de turismo, donde el $66.45 \%$ del total de matriculados son mujeres. De este modo, en esta muestra, el $32.3 \%$ de los encuestados son hombres frente a un $67.7 \%$ mujeres. La tabla 1 presenta sus características por género, edad, curso, experiencia profesional previa y participación en asociaciones de carácter social, cultural o deportivo. El nivel de significación de la muestra es del $95 \%$, siendo su error muestral del $3.76 \%$ sobre el total del alumnado de turismo.

Tabla 1. Características de la muestra por género

\begin{tabular}{|c|c|c|c|c|c|}
\hline Edad & Hombres & Mujeres & Curso & Hombres & Mujeres \\
\hline$<20$ años & $16.96 \%$ & $28.51 \%$ & $1^{\circ}$ & $23.21 \%$ & $34.47 \%$ \\
\hline 20-25 años & $69.64 \%$ & $67.66 \%$ & $2^{o}$ & $27.68 \%$ & $25.11 \%$ \\
\hline \multirow[t]{2}{*}{$>25$ años } & $13.39 \%$ & $3.83 \%$ & $3^{\circ}$ & $16.96 \%$ & $16.17 \%$ \\
\hline & & & $4^{\circ}$ & $32.14 \%$ & $24.26 \%$ \\
\hline $\begin{array}{l}\text { Experiencia } \\
\text { profesional }\end{array}$ & Hombres & Mujeres & $\begin{array}{l}\text { Participación } \\
\text { asociaciones }\end{array}$ & Hombres & Mujeres \\
\hline $\mathrm{Si}$ & $49.11 \%$ & $34.04 \%$ & $\mathrm{Si}$ & $44.64 \%$ & $31.91 \%$ \\
\hline No & $50.89 \%$ & $65.96 \%$ & No & $55.36 \%$ & $68.09 \%$ \\
\hline
\end{tabular}

Fuente: Elaboración propia. 


\section{Instrumentos}

Por la naturaleza de la pregunta de investigación, se ha optado por dar a la investigación un enfoque cuantitavo, con el objeto de medir el grado de intensidad de los factores previamente mencionados en la intención emprendedora. La herramienta de investigación utilizada ha sido un trabajo de campo consistente en la realización de un cuestionario sobre la actitud hacia el emprendimiento, adecuado al objeto de este estudio, tomando como referencia las investigaciones de Genesca y Veciana (1984), contrastado por Aponte (2002) y Díaz, et al. (2007). Inicialmente se realizó un pretest a 9 estudiantes para comprobar su validez, perfilando algunas expresiones que se determinaron poco o no adecuadas.

\section{Procedimiento}

Se ha empleado un muestreo de conveniencia, ordinariamente utilizado en este tipo de investigaciones donde los encuestados están disponibles en un espacio y tiempo determinado. En un primer momento, una vez seleccionado y adaptado el cuestionario para el propósito del estudio, se elaboró un documento sobre la motivación del estudio que fue remitido al coordinador de Turismo. Tras obtener autorización, se contactó con los responsables de las asignaturas para que facilitaran su ayuda para poder pasar los cuestionarios entre el alumnado. El investigador tenía la advertencia de poder realizar aclaraciones de significado de conceptos, pero ninguna otra aclaración sobre la formulación del cuestionario.

\section{Análisis Estadístico}

Como un paso preliminar, se ha recurrido a un análisis descriptivo bivariante que permita conocer si dos variables están asociadas. La herramienta por la que se ha optado es la tabla de contigencia. Por otra parte dos estadísticos diferentes han sido utilizados para medir la existencia o no de relación. En el caso de que las variables, que se comparaban entre sí, fuesen ambas dicotómicas en la forma $2 \times 2$ el estadístico empleado ha sido el Coeficiente Phi de Pearson (en adelante $\phi$ ); en segundo lugar para el caso en que el número de filas fuese diferente al de columnas se ha optado por el coeficiente de contingencia de Pearson (en adelante $\left.\chi^{2}\right)$.

Como aparato estadístico principal se ha empleado un análisis de regresión logística (Fuentes \& Sánchez, 2010; Turró, Urbano \& Peris-Ortiz., 2014) para determinar las competencias intrapersonales que afectan a la actitud emprendedora de los estudiantes. Por otra parte, también se estudiaron dos factores que hemos denominado socioculturales y dos de 
formación, como variables moderadoras de la intención emprendedora. Para una mayor aproximación se ha construido un modelo por género con el objeto de comprobar si las variables significativas que determinan el emprendimiento varían. Para el análisis estadístico de los datos se ha utilizado el paquete estadístico SPSS.

\section{Resultados}

En primer lugar se comprobó si existía relación entre las variables género del encuestado por un lado y el desempeño de otras actividades como la laboral o la pertenencia a algún tipo de organización. El Coeficiente Phi de Pearson entre las variables género y experiencia laboral tiene un valor de $\phi(1, N=347)=.140, p<.05$, además se detectó la existencia de relación entre género y la participación en algún tipo de asociación $\phi(1, N=347)=.143, p<.05$.

\section{Deseabilidad de emprendimiento}

La segunda cuestión planteada trata sobre la deseabilidad de crear una empresa propia. En este sentido, el alumnado de la enseñanza superior de turismo tiene una postura favorable al emprendimiento (73.6\%), pero esta propensión inicial es más intensa entre los alumnos (78\%) que entre las alumnas $(69.8 \%), \phi(1, N=347)=.085, p<.1$, como los resultados de Díaz et al. (2007). Además, aquellos que realizan alguna actividad laboral, o cuentan con alguna experiencia profesional previa, $\phi(1, N=347)=.194, p<.01$, tienen mayor deseo de emprendimiento $(84.1 \%)$ que los estudiantes sin experiencia previa (66.7\%). Por otra parte, el estar finalizando los estudios favorece la deseabilidad hacia el emprendimiento, $\chi^{2}$ (1, $N=347)=.247, p<.01$, con un $79.2 \%$ y $87.2 \%$ respectivamente para el tercer y cuarto curso, frente a un $57.9 \%$ de alumnos que cursan primero y un $71.7 \%$ de segundo. A pesar de este elevado porcentaje que manifiestan su deseo de autoempleo, existe un importante número que percibe que existe una gran dificultad en emprender (76.9\%), no detectándose diferencias apreciables en función del sexo, edad, experiencia profesional, existencia de empresarios en la familia, año cursado y participación en algún tipo de asociación. La edad también es un condicionante a la hora de la deseabilidad de emprender, $\chi^{2}(1, N=347)=.112, p<.1$. En este caso existe una diferencia de 5 puntos porcentuales a favor de los estudiantes de mayor edad. Al profundizar sobre la intención futura de crear o no una empresa, lo que la literatura ha venido a denominar emprendedores potenciales (Huefner, Hunt, \& Robinson, 1996; López, García, Cano, Gea \& de la Fuente, 2010), la tasa de respuesta de los estudiantes baja hasta el 33.3\%. 
Percepción de las competencias intrapersonales

En el cuestionario se pidió una valoración de sí mismos sobre un conjunto de competencias. Con esta finalidad se utilizó una escala Likert de 4 puntos, correspondiéndose el valor 1 a "escasez de la competencia" y 4 "alta presencia de la competencia", con el objetivo de determinar si existen diferencias por sexo. La herramienta estadística de contraste empleada fue la prueba no paramétrica de U de Mann-Whitney.

De los datos obtenidos se intuye que existen diferencias significativas en la percepción de mujeres y varones en 3 de las 20 competencias analizadas (tabla 2). Los estudiantes de género masculino tienen puntuaciones superiores en las competencias " 2 " y "20", se trata de aspectos relacionados con la iniciativa, la autoconfianza, el optimismo y predisposición hacia las actividades más complejas, como competencias asociadas al perfil emprendedor (Boydston Hopper \& Wright, 2000; Davidsson, 1989; McClelland, 1961). Por el contrario, las estudiantes valoran más positivamente la competencia “3” relacionada con la responsabilidad y la disciplina. De este modo, se consideran que poseen un mayor rigor a la hora de trabajar, dedicar tiempo a obtener buenos resultados y la motivación hacia el estudio (Echavarri, Godoy \& Olaz, 2007; Edel, 2003; Hackett \& Betz, 1989; Olaz, 2003).

Tabla 2. Contraste U Mann-Whitney competencias intrapersonales-sexo

\begin{tabular}{lccl}
\hline Competencias intrapersonales & $\begin{array}{c}\text { Media } \\
\text { hombres }\end{array}$ & $\begin{array}{c}\text { Media } \\
\text { mujeres }\end{array}$ & $\begin{array}{c}\text { Mann-Whitney } \\
\text { Valor Z (sig.) }\end{array}$ \\
\hline 1. Adaptabilidad a cambios & 3.34 & 3.37 & $-.117(.907)$ \\
2. Autoconfianza & 3.04 & 2.81 & $-1.744(.081)^{*}$ \\
3. Autodisciplina & 3.09 & 3.32 & $-1.785(.074)^{*}$ \\
4. Autonomía en el trabajo & 3.38 & 3.26 & $-.601(.548)$ \\
5. Prever y anticipar & 3.28 & 3.08 & $-1.247(.212)$ \\
6. Facilidad para la comunicación & 3.22 & 3.34 & $-.466(.641)$ \\
7. Creatividad para resolver problemas & 2.91 & 2.93 & $-.091(.928)$ \\
8. Curiosidad por conocer & 3.43 & 3.43 & $-.021(.983)$ \\
9. Dedicación en el trabajo & 3.02 & 3.17 & $-1.189(.235)$ \\
10. Optimismo ante dificultades & 2.98 & 2.73 & $-1.64(.101)$ \\
11. Estabilidad emocional & 3.28 & 3.10 & $-1.154(.248)$ \\
12. Perseverante & 3.17 & 3.13 & $-.046(.963)$ \\
13. Entusiasta para iniciar proyectos & 3.15 & 3.19 & $-.376(.707)$ \\
14. Tolerancia ante fracasos & 2.72 & 2.72 & $-.009(.993)$ \\
15. Iniciativa en situaciones complejas & 2.89 & 2.84 & $-.404(.686)$ \\
16. Imaginación en la evolución de proyectos & 3.28 & 3.25 & $-.277(.782)$ \\
17. Gusto por la independencia & 3.50 & 3.38 & $-.96(.337)$ \\
18. Estímulo ante nuevos retos & 3.23 & 3.12 & $-.728(.467)$ \\
19. Responsabilidad ante decisiones & 3.45 & 3.50 & $-.759(.448)$ \\
20. Predisposición para asumir riesgos & 3.07 & 2.80 & $-2.089(.037)^{* *}$ \\
\hline
\end{tabular}

Nota: * $p<.1 * * p<.05 ; * * * p<.01$. 


\section{Determinantes de la intención emprendedora}

Se han planteado los siguientes modelos logit binarios:

- Modelo 1: Intención emprendedora dependiente de las competencias intrapersonales.

- Modelo 2: Intención emprendedora dependiente de las competencias intrapersonales y ambiente sociocultural (experiencia profesional y tradición empresarial en la familia).

- Modelo 3: Intención emprendedora dependiente de las competencias intrapersonales, ambiente sociocultural y de formación (participación en algún tipo de asociación y curso).

La variable dependiente toma como cuestión la intencionalidad o no del autoempleo. La variable se codifica tomando únicamente dos valores, por un lado el valor 0 (no hay intención o la intención es vaga) y por el otro el valor 1 (sí hay intención). Las variables independientes se han recodificado como variables dicotómicas con el valor 0 , presencia reducida de la competencia y 1 , presencia alta de la competencia. Lo mismo ocurre con las variables moderadoras.

Siendo los modelos:

$\mathrm{Y}_{\mathrm{i}}($ hombre/mujer $)=\beta+1$ competen_1 $1_{\mathrm{i}}+\ldots+$ 20competen_20 $0_{\mathrm{i}}+21$ exper_prof $\mathrm{i}_{\mathrm{i}}+$ 22empres_famil $1_{i}+23$ curso +24 partic_asociac $_{i}+e_{i}$

Se realizó un análisis de regresión por la metodología de estimación por pasos (razón de verosimilitud) ante la posible existencia de multicolinealidad. Los modelos finales presentados sólo incluyen variables que han resultado significativas.

Tabla 3. Modelo logit binario sobre intención emprendedora. Hombres

\begin{tabular}{|c|c|c|c|c|c|c|c|c|c|}
\hline \multirow[b]{2}{*}{ Competencias } & \multicolumn{3}{|c|}{ Modelo 1} & \multicolumn{3}{|c|}{ Modelo 2} & \multicolumn{3}{|c|}{ Modelo 3} \\
\hline & $\beta$ & Coefic & Signific & $\beta$ & Coefic & Signific & $\beta$ & Coefic & Signific \\
\hline 1. Adaptabilidad & -1.199 & 5.85 & $.016 * *$ & -1.039 & 4.917 & $.027 * *$ & -14.436 & 5.010 & $.025 * *$ \\
\hline 2. Autoconfianza & & & & & & & & & \\
\hline 3. Autodisciplina & 1.223 & 5.904 & $.015^{* *}$ & 1.405 & 6.767 & $.009 * * *$ & 5.575 & 5.648 & $.017 * *$ \\
\hline $\begin{array}{l}\text { 4. Autonomía en el } \\
\text { trabajo }\end{array}$ & & & & & & & 6.221 & 5.565 & $.018 * *$ \\
\hline 5. Prever y anticipar & & & & & & & -13.999 & 5.352 & $.021 * *$ \\
\hline $\begin{array}{l}\text { 6. Facilidad para la } \\
\text { comunicación } \\
\text { 7. Creatividad para } \\
\text { resolver problemas }\end{array}$ & & & & & & & 12.212 & 5.937 & $.015^{* *}$ \\
\hline $\begin{array}{l}\text { 8. Curiosidad por cono- } \\
\text { cer }\end{array}$ & & & & & & & 15.257 & 5.734 & $.017 * *$ \\
\hline
\end{tabular}




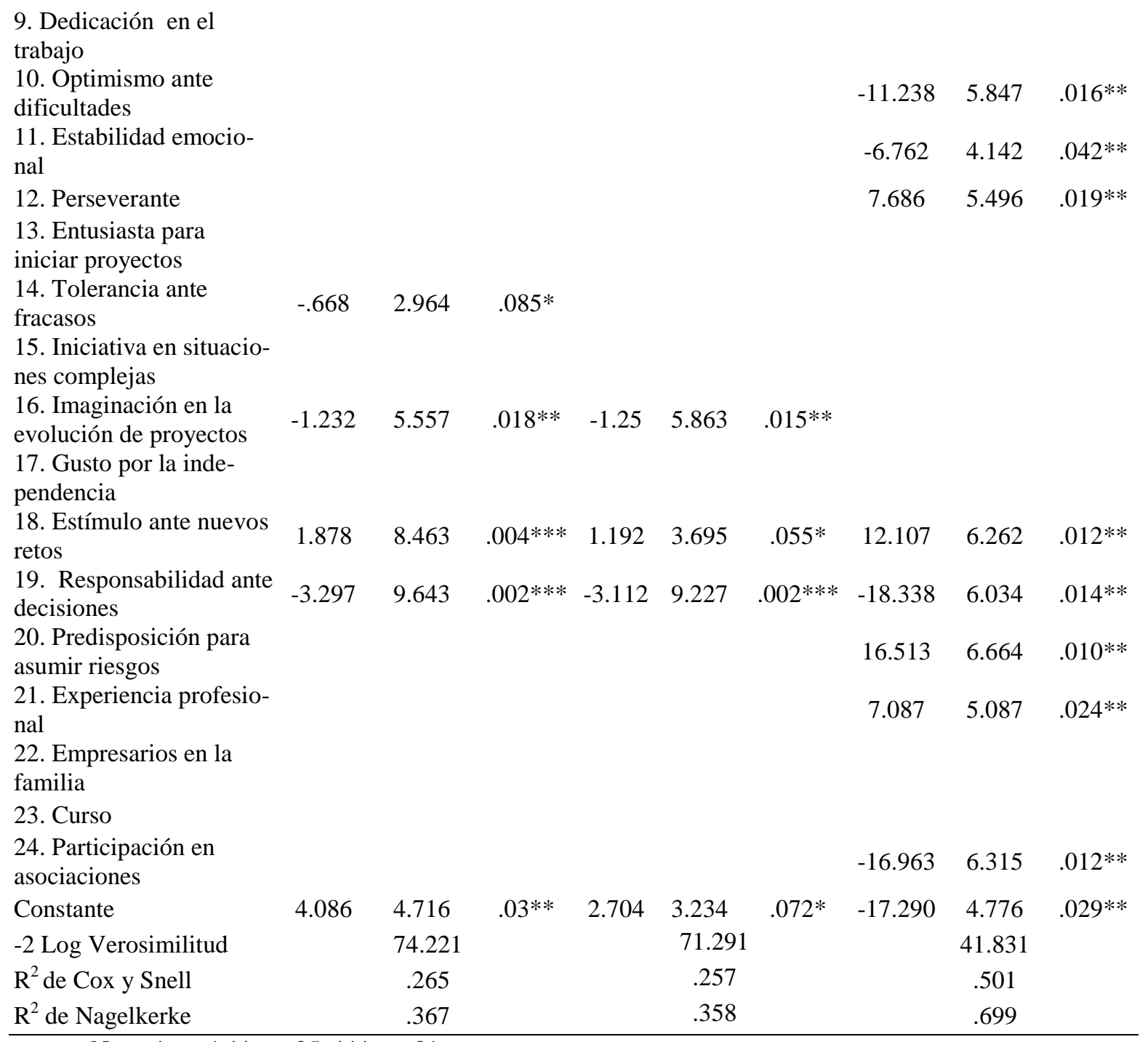

En el modelo 1 de los chicos (tabla 3), se observa la presencia de competencias intrapersonales significativas como la autodisciplina en el trabajo y estímulo ante nuevos retos, atributos relacionados con la constancia y la creación, aunque este modelo tiene un bajo nivel explicativo. La introducción de variables moderadoras de experiencia profesional y participación en asociaciones del modelo 3, ha permitido incrementar el número de atributos que determinan el emprendimiento y mejorar sensiblemente el grado explicativo del modelo predictivo sobre la actitud emprendedora de los hombres, aumentando hasta el 0.7 aproximadamente el $\mathrm{R}^{2}$ corregido. 
Tabla 4. Modelo logit binario sobre intención emprendedora. Mujeres

\begin{tabular}{|c|c|c|c|c|c|c|c|c|c|}
\hline \multirow[b]{2}{*}{ Competencias } & \multicolumn{3}{|c|}{ Modelo 1} & \multicolumn{3}{|c|}{ Modelo 2} & \multicolumn{3}{|c|}{ Modelo 3} \\
\hline & $\beta$ & Coefic. & Signific. & $\beta$ & Coefic. & Signific. & $\beta$ & Coefic. & Signific \\
\hline \multicolumn{10}{|l|}{ 1. Adaptabilidad } \\
\hline \multicolumn{10}{|l|}{ 2. Autoconfianza } \\
\hline 3. Autodisciplina & & & & -.148 & .169 & .681 & & & \\
\hline $\begin{array}{l}\text { 4. Autonomía en el } \\
\text { trabajo }\end{array}$ & -.487 & 3.636 & $.057^{*}$ & -.749 & 4.601 & $.032 * *$ & -.804 & 3.496 & $.062 *$ \\
\hline \multicolumn{10}{|l|}{ 5. Prever y anticipar } \\
\hline \multicolumn{10}{|l|}{$\begin{array}{l}\text { 6. Facilidad para la } \\
\text { comunicación }\end{array}$} \\
\hline \multicolumn{10}{|l|}{$\begin{array}{l}\text { 7. Creatividad para } \\
\text { resolver problemas }\end{array}$} \\
\hline \multicolumn{10}{|l|}{$\begin{array}{l}\text { 8. Curiosidad por } \\
\text { conocer }\end{array}$} \\
\hline $\begin{array}{l}\text { 9. Dedicación en el } \\
\text { trabajo }\end{array}$ & -.626 & 6.763 & $.009^{*}$ & -.922 & 6.847 & $.009^{* * *}$ & -1.030 & 8.278 & $.004 * * *$ \\
\hline \multicolumn{10}{|l|}{$\begin{array}{l}\text { 10. Optimismo ante } \\
\text { dificultades }\end{array}$} \\
\hline $\begin{array}{l}\text { 11. Estabilidad emocio- } \\
\text { nal }\end{array}$ & .715 & 9.088 & $.003^{* * * *}$ & .966 & 8.238 & $.004 * * *$ & .795 & 5.072 & $.024 * *$ \\
\hline 12. Perseverante & & & & -.998 & 5.336 & $.021 * *$ & -.891 & 4.861 & $.027 * *$ \\
\hline \multicolumn{10}{|l|}{$\begin{array}{l}\text { 13. Entusiasta para } \\
\text { iniciar proyectos }\end{array}$} \\
\hline \multicolumn{10}{|l|}{$\begin{array}{l}\text { 14. Tolerancia ante } \\
\text { fracasos }\end{array}$} \\
\hline \multicolumn{10}{|l|}{$\begin{array}{l}\text { 15. Iniciativa en situa- } \\
\text { ciones complejas }\end{array}$} \\
\hline \multicolumn{10}{|l|}{$\begin{array}{l}\text { 16. Imaginación en la } \\
\text { evolución de proyectos }\end{array}$} \\
\hline \multicolumn{10}{|l|}{$\begin{array}{l}\text { 17. Gusto por la inde- } \\
\text { pendencia }\end{array}$} \\
\hline \multicolumn{10}{|l|}{$\begin{array}{l}\text { 18. Estímulo ante } \\
\text { nuevos retos }\end{array}$} \\
\hline \multicolumn{10}{|l|}{$\begin{array}{l}\text { 19. Responsabilidad } \\
\text { ante decisiones }\end{array}$} \\
\hline \multicolumn{10}{|l|}{$\begin{array}{l}\text { 20. Predisposición para } \\
\text { asumir riesgos }\end{array}$} \\
\hline $\begin{array}{l}\text { 21. Experiencia profe- } \\
\text { sional }\end{array}$ & & & & 2.316 & 15.189 & $.000^{* * *}$ & 2.720 & 13.747 & $.000 * * *$ \\
\hline $\begin{array}{l}\text { 22. Empresarios en la } \\
\text { familia }\end{array}$ & & & & -1.014 & 4.262 & $.039 * *$ & -0.951 & 3.796 & $.050 *$ \\
\hline \multicolumn{10}{|l|}{ 23. Curso } \\
\hline \multicolumn{10}{|l|}{$\begin{array}{l}\text { 24. Participación en } \\
\text { asociaciones }\end{array}$} \\
\hline Constante & -0.109 & 0.026 & 0.872 & -0.182 & .024 & .877 & -1.968 & 1.431 & .232 \\
\hline -2 Log Verosimilitud & & 190.475 & & & 155.390 & & & 150.094 & \\
\hline $\mathrm{R}^{2}$ de Cox y Snell & & 0.123 & & & 0.265 & & & 0.267 & \\
\hline $\mathrm{R}^{2}$ de Nagelkerke & & 0.170 & & & 0.367 & & & 0.371 & \\
\hline
\end{tabular}

Nota: $* p<.1 * * p<.05 ; * * * p<.01$. 
En el caso de las chicas (tabla 4), los modelos explicativos tienen un bajo poder explicativo global ( $\mathrm{R}^{2}$ de 0.170 para el modelo 1 , aumentando hasta 0.371 para el modelo 3 ), con pocas competencias intrapersonales explicativas. La experiencia profesional $(\mathrm{p}<.01)$ presenta un coeficiente positivo asociado, al igual que en el modelo de los chicos, siendo un factor de éxito para la exploración de nuevas oportunidades de negocio (Fayolle \& Gailly, 2004; Lazear, 2004). Por su parte, no se puede decir lo mismo de la presencia de empresarios en la familia. Al revés, parece que la presencia de emprendedores en la misma y su ejemplo, afecta negativamente a la intención emprendedora. Esta desafección, contrario a los trabajos de Basu \& Goswami (1999) y de Duchesneau \& Gartner (1990) puede venir causada por una percepción generalizada de un menor grado de viabilidad de las pequeñas y medianas empresas derivadas de los efectos adversos de la reciente crisis económica.

\section{Discusión y Conclusiones}

El principal objetivo de este estudio era conocer las competencias intrapersonales que determinan el perfil emprendedor de los alumnos en la educación superior de turismo y determinar si existen diferencias significativas según género de las que se derivan tres preguntas de investigación enunciadas en este trabajo. En referencia a la primera cuestión de la investigación (RQ1), como un inciso previo, los resultados indican que los alumnos varones presentan mayor predisposición a compaginar su actividad académica con alguna actividad de tipo profesional y, además, tienen un mayor grado de participación en otras actividades de tipo cultural, deportivo o social, independiente de las actividades laborales previamente comentadas, que sus compañeras. Centrándonos en la cuestión sobre la deseabilidad de crear su propia empresa, existe una intensa propensión inicial entre del alumnado en general, aunque en mayor proporción entre los alumnos que las alumnas. También, el hecho de que los alumnos encuestados que dijeron tener experiencia profesional previa tengan mayor deseo de emprendimiento, nos hace sospechar que el menor deseo de emprendimiento femenino que se ha detectado, puede estar influenciado por la menor experiencia profesional previa que afirman tener las alumnas en sus contestaciones. Además, los denominados emprendedores potenciales presentan una tasa de respuesta baja, el 33.3\%. Con estos primeros indicios se puede afirmar que el autoempleo es una alternativa factible de llevar a la práctica para el alumnado de educación superior de turismo, aunque se pone de relieve una cierta contradicción entre el deseo manifiesto mayoritario de creación de una empresa propia, y una verdadera acción que lleve a materializar la idea inicial. 
En referencia a la segunda cuestión de la investigación (RQ2), no se aprecian diferencias significativas importantes por género en las veinte competencias intrapersonales analizadas que tienen relación con el emprendimiento. Aunque los alumnos presentan un mayor nivel de autoconfianza y de predisposición a asumir riesgos, frente a las alumnas que tienen una mayor capacidad de autodisciplina. Pero los resultados del modelo 1, donde se analiza la intención emprendedora dependiente únicamente de las competencias intrapersonales, han resultado poco significativos en ninguno de los dos sexos.

Sobre la tercera cuestión de la investigación (RQ3), los resultados del modelo 3 nos permiten intuir la importancia que tiene para los alumnos ser activos tanto social como profesionalmente, bien a través de asociaciones culturales, deportivas, etc., o mediante trabajos o prácticas, como estímulo de aquellas competencias necesarias para el autoempleo y el descubrimiento de oportunidades de negocio. Sin embargo, resulta interesante comprobar que para el caso de las alumnas, aunque sigue teniendo importancia la experiencia profesional, no hay ninguna variable de competencia significativa relacionada con el riesgo o la iniciativa, y sí con las capacidades relacionadas con el trabajo sacrificado y la abnegación, aunque influyen negativamente en la intención emprendedora, además de tener un modelo poco explicativo. De todas las competencias significativas que explican la intención emprendedora, sólo existe una coincidencia entre ambos sexos, la estabilidad emocional que hace referencia a la madurez y al equilibrio de la persona.

La incorporación de estudios sobre el emprendimiento que se centren en el género, debe de servir como herramienta que ayude a la elaboración de políticas educativas donde se fomente, no sólo el acceso de la mujer a puestos de trabajo por cuenta ajena, sino a través de la creación de autoempleo (Bruton et al., 2010; De Bruin et al., 2007). El importante peso que tienen las mujeres dentro de la formación superior en turismo no se corresponde con una similar propensión hacia el emprendimiento, como se ha demostrado en este estudio. Existen trabajos que afirman que existe una brecha de género notable a favor de los hombres cuando se habla de emprendimiento (Klyver, Suna \& Rostgaard, 2013; Kreide, 2003). Pero las estrategias de educación desde la niñez, pueden ser alentadoras o desalentadoras de emociones y se han relacionado con aspectos de la competencia emocional (Gottman, Katz \& Hooven, 1997). La mayor protección que reciben las chicas en el entorno familiar, fomenta la dependencia, la obediencia y la falta de seguridad en sí mismas (Zahn-Waxler, 2000), por lo que frena el estímulo ante nuevos retos y su predisposición a asumir riesgos, que es básico en el empren- 
dimiento. Es necesario implementar políticas educativas específicas para las mujeres que fomenten desde la educación primaria hasta la superior, éstas y otras competencias relacionadas con el emprendimiento. También es fundamental que las alumnas y alumnos tengan, en sus planes de estudio, periodos amplios de prácticas en empresas, porque es una variable determinante, en ambos géneros, hacia el emprendimiento como se ha demostrado en esta investigación.

La limitación principal de este trabajo está relacionada con la especificidad de la muestra, al centrarse en los Estudios Superiores de Turismo de la Universidad de Córdoba (España), por lo que puede causar sesgo sobre alguna competencia de los encuestados y presentar problemas de generalización en la actitud hacia el emprendimiento. A pesar de esta limitación, los resultados y conclusiones de esta investigación hacen aportaciones originales a la literatura, proporcionando una mejor comprensión de la llamada brecha de género en el emprendimiento, en perjuicio de la mujer.

\section{Referencias}

Alburquerque, F. (2008). Innovación, transferencia de conocimientos y desarrollo económico territorial: una política pendiente. Arbor, 184(732), 687-700. Doi: 10.3989/arbor.2008 .1732 .215 .

Ang, S. H., \& Hong, D. G. P. (2000). Entrepreneurial spirit among East Asian Chinese. Thunderbird International Business Review. 42 (3), 285-309. Doi: 10.1002/ 15206874(200005/06)42:3<285::AID-TIE2>3.0.CO;2-5

Aponte, M. (2002). Factores condicionantes de la creación de empresas en Puerto Rico: Un enfoque institucional. Tesis Doctoral. Universidad Autónoma de Barcelona. Disponible en: http://www.tdx.cat/bitstream/handle/10803/3946/mag1de1.pdf ?sequence=1.

Basu, A., \& Goswami, A. (1999). Determinants of South Asian entrepreneurial growth in Britain: A multivariate analysis. Small Business Economics, 13, 57-70. Doi: 10.1023 /A:1008025628570.

Begley, T. M., \& Boyd, D.P. (1987). A comparison of entrepreneurs and managers of small business firms. Journal of Management, 13(1), 99-108. Doi: 10.1177/014920638701 300108.

Benavides, M. M., Sánchez-García, M. I., \& Luna-Arocas, R. (2004). El proceso de aprendizaje para los emprendedores en la situación actual: un análisis cualitativo en el ámbito 
universitario. Dirección y Organización, DyO, 30, 30-48. Disponible en: http://www.revistadyo.com/index.php/dyo/article/view/122/122.

Blake, A., Sinclair, M. T., \& Soria, J. A. C. (2006). Tourism productivity-evidence from the United Kingdom. Annals of Tourism Research, 33(4), 1099-1120. Doi: 10.1016/ j.annals.2006.06.001

Boydston, M., Hopper, L., \& Wright, A. (2000). Locus of control and entrepreneurs in a small town: San Antonio, TX: Proceedings of ASBE. Disponible en: https://drive.google.com/file/d/0B9pflhVOKMWBYmpNSkxxbXJvc2c/view.

Bruton, G. D., Ahlstrom, D., \& Li, H.-L. (2010). Institutional Theory and Entrepreneurship: Where Are We Now and Where Do We Need to Move in the Future? Entrepreneurship Theory and Practice, 34, 421-440. Doi: 10.1111/j.1540-6520.2010.00390.x.

Caliendo, M., Fossen, F. M., \& Kritikos, A. S. (2009). Risk attitudes of nascent entrepreneurs-new evidence from an experimentally validated survey. Small Business Economics, 32(2), 153-167. Doi: 10.1007/s11187-007-9078-6.

Castro, J., Barrenechea, J., \& Ibarra, A. (2011). Cultura emprendedora, innovación y competencias en la educación superior. El caso del Programa GAZE. ARBOR, 187(3), 207212. Doi: 10.3989/arbor.2011.Extra-3n3146.

Cawley, M., \& Gillmor, D. A. (2008). Integrated rural tourism: Concepts and practice. Annals of Tourism Research, 35(2), 316-337. Doi: 10.1016/j.annals.2007.07.011.

Danilo, É., Melo, M. C., \& Rocha, A. L. (2012). El turismo en la sociedad de la información. Un abordaje conceptual sobre el "pos-turismo". Estudios y Perspectivas en Turismo, 21(5) 1262-1280. Disponible en: http://www.redalyc.org/articulo.oa?id= 180724156011.

Davidsson, P. (1989). Continued Entrepreneurship and Small Firm Business. Stockholm School of Economics: Stockholm.

De Bruin, A., Brush, C. G., \&Welter, F. (2007). Advancing a framework for coherent research on women's entrepreneurship. Entrepreneurship Theory and Practice, 31(3), 323-340. Doi: 10.1111/j.1540-6520.2007.00176.x

de la Fuente, J., Vera, M. M., \& Cardelle-Elawar, M. (2012). Aportaciones de la Psicología de la Innovación y del Emprendimiento a la Educación, en la Sociedad del Conocimiento. Electronic Journal of Research in Educational Psychology, 10(28), 941-966. Disponible en: http://www.investigacion-psicopedagogica.org/revista/new/ english/ContadorArticulo.php?772. 
Delmar, F., \& Davidsson, P. (2000). Where do they come from? Prevalence and characteristics of nascent entrepreneurs. Entrepreneurship and Regional Development, 12, 1-23. Doi: $10.1080 / 089856200283063$.

Detienne, D., \& Chandler, G. (2004). Opportunity Identification and its role in the Entrepreneurial Classroom: A Pedagogical Approach and Empirical Test. Academy of Management Learning and Education, 3(3), 242-257. Doi: 10.5465/AMLE.2004. 14242103.

Díaz, J. C., Hernández, R. M., \& Barata, M. L. (2007). Estudiantes universitarios y creación de empresas. Un análisis comparativo entre España y Portugal. In: Ayala, J. C. (coord.), Conocimiento, innovación y emprendedores: camino al futuro (pp. 13381355). Madrid: Grupo FEDRA. Disponible en: http://dialnet.unirioja.es/servlet /articulo? codigo $=2234363$.

Díaz, J. C., \& Jiménez J. J. (2004). Influencia del género en la actividad empresarial. Diferencias en los resultados y factores de éxito. XVI Congreso ACEDE, Murcia. Díaz, J. C., Sánchez, M. C. \& Postigo, M. V. (2007). Mujer y creación de empresas en Extremadura: un análisis comparativo. In: Ayala, J. C. (coord.), Conocimiento, innovación y emprendedores: camino al futuro (pp. 1180-1198). Madrid: Grupo FEDRA. Disponible en: dialnet.unirioja.es/descarga/articulo/2233340.pdf.

Duchesneau, D. A., \& Gartner, W. B. (1990). A profile of new venture success and failure in an emerging industry. Journal of Business Venturing, 5 (5), 297-312. Doi: 10.1016/0883-9026(90)90007-G.

Echavarri, M., Godoy, J. C., \& Olaz, F. (2007). Diferencias de género en habilidades cognitivas y rendimiento académico en estudiantes universitarios. Universitas Psychologica, 6 (2), 319-329. Disponible en: http://pepsic.bvsalud.org/scielo. php?script=sci_arttext\&pid=S 1657-926720070002000 11\&lng=pt\&tlng=es.

Edel, R. (2003). El rendimiento académico, concepto investigación y desarrollo. REICE, Revista Electrónica Iberoamericana sobre Calidad, Eficacia y Cambio en Educación, 1. Disponible en: http://www.ice.deusto.es/RINACE/reice/vol1n2/Edel.pdf.

Elkington, J., \& Hartigan, P. (2008). The power of unreasonable people. How Social Entrepreneurs Create Markets that Change the World. Boston: Harvard Business School Publishing.

Espelt, N. G. (2009). Retos para el sistema educativo en turismo. Actas del XIV Congreso AECIT. Gijón 18-20 /10/2009, 211-219. Disponible en: http://www.aecit.org/actas/ gijon /XIV_Congreso_AECIT_0.DocCompleto.pdf. 
Evans, D. S., \& Leighton, L. S. (1989). Some Empirical Aspects of Entrepreneurship. The American Economic Review, 79 (3), 519-535. Disponible en: http://www.jstor.org/ stable/1806861.

Fayolle, A., \& Gailly, B. (2004). Using the theory of planned behaviour to assess entrepreneurship teaching programs: a first experimentation. Paper presented at the IntEnt2004 ConferenceNapoli, Italy: July.

Fuentes, F. J., \& Sánchez, S. (2010). Análisis del perfil emprendedor: una perspectiva de género. Estudios de Economía Aplicada, 28 (3), 1-28. Disponible en: http://www.revista-eea.net/documentos/28306.pdf.

Genesca, E., \& Veciana, J. M. (1984). Actitudes hacia la creación de empresas. Información Comercial Española, 611, 147-155.

Getz, D., \& Carlsen, J. (2005). Family business in tourism - state of the art. Annals of Tourism Research, 32(1), 237-258. Doi: 10.1016/j.annals.2004.07.006.

González-Morales, M. O. (2001). Actitudes y motivaciones hacia el trabajo y la creación de empresas según género. Boletín Económico ICE, 2709, 21-29. Disponible en: http://www.revista-eea.net/documentos/28306.pdf.

Gottman, J. M., Katz, L. F., \& Hooven, C. (1997). Meta-emotion: How families communicate emotionally. Hillsdale, NJ: Erlbaum

Gurel, E., Altinay, L., \& Daniele, R. (2010).Tourism students' entrepreneurial intentions. Annals of Tourism Research, 37(3), 646-669. Doi: 10.1016/j.annals.2009.12.003.

Gürol, Y., \& Atsan, N. (2006). Entrepreneurial characteristics amongst university students: some insights for entrepreneurship education and training in Turkey. Education and Training, 48(1), 25-38. Doi: http://dx.doi.org/10.1108/00400910610645716.

Hackett, G., \& Betz, N. E. (1989). An Exploration of the Mathematics Selfefficacy/mathematics Performance Correspondence. Journal for Research in Mathematics Education, 20, 261-273. Doi: 10.2307/749515

Holmgren, C., \& From, J. (2005). Taylorism of the mind: Entrepreneurship education from a perspective of educational research. European Educational Research Journal, 4(4), 382-390. Doi:10.2304/eerj.2005.4.4.4.

Huefner, J. C., Hunt, H. K., \& Robinson, P. B. (1996). A comparison of four scales predicting entrepreneurship. Academy of Entrepreneurship Journal, 1, 56-80.

Janney, J. J., \& Dess, G. G. (2006). The risk concept for entrepreneurs reconsidered: new challenges to the conventional wisdom. Journal of Business Venturing, 21, 385-400. Doi: $10.1177 / 0266242614542853$. 
Klyver, K., Suna N., \& Rostgaard M. (2013). Women's self-employment: An act of institutional (dis)integration? A multilevel, cross-country study. Journal of Business Venturing, 28, 474-488.

Koh, H. C. (1996). Testing hypotheses of entrepreneurial characteristics: a study of Hong Kong MBA students. Journal of Managerial Psychology, 11(3), 12-25. Doi: http://dx.doi.org/10.1108/02683949610113566.

Kreide, R. (2003). Self-employment of women and welfare-state policies. International Review of Sociology, 13(1), 205-218. Doi: 10.1080/0390670032000087069.

Lazear, E. P. (2004). Balanced skills and entrepreneurship. American Economic Review, 94 (2), 208-211. Doi: 10.1257/0002828041301425.

López J., \& García J. (2011). Optimismo, pesimismo y realismo disposicional en emprendedores potenciales de base tecnológica. Psicothema, 23(4), 611-616.

López, J., García, J., Cano, C. J., Gea, A. B., \& De la Fuente, L. (2010). A definition of potential entrepreneur from a probabilistic point of view. In M. J. Blanca, R. Alarcón y D. López-Montiel (Coords.), Actas del XI Congreso de Metodología de las Ciencias Sociales y de la Salud (pp. 577-581). UMA: Tecnolex. Disponible en: http://www.jpuga.es /Publicaciones/Congresos/prob_def_pot_entrep.pdf. McClelland, D. C. (1961). The Achieving Society. Princeton, NJ: D. Van Norstrand Co.

Meek, W. R., Pacheco, D. F., \& York, J. G. (2010). The impact of social norms on entrepreneurial action: evidence from the environmental entrepreneurship context. Journal of Business Venturing, 25, 493-509. Doi:10.1016/j.jbusvent.2009.09.007.

Mueller, S. L. (2004). Gender gaps in potential for entrepreneurship across countries and cultures. Journal of Developmental Entrepreneurship, 9(3), 199-220.

Nga, H. K. J., \& Shamuganathan, G. (2010). The influence of personality traits and demographic factors on social entrepreneurship start up intentions. Journal of Business Ethics, 95(1), 259-260. Doi 10.1007/s10551-009-0358-8.

Olaz, F. O. (2003). Autoeficacia y variables vocacionales. Psicología Educativa, 9 (1), 5-14.

Pedreño, A., \& Ramón, A (2009). El turismo: globalización, competitividad y sostenibilidad. En Colección Mediterráneo Económico: "El futuro de la economía española". Vol. 16, Coordinador: Velarde Fuertes, J. CAJAMAR. Disponible en: http://www. publicacionescajamar.es/pdf/publicacionesperiodicas/mediterraneoeconomico/16/16-281.pdf.

Shapero, A., \& Sokol, L. (1982). The Social Dimensions of Entrepreneurship. In C. A. Kent, D. L. Sexton y K. H. Vesper, Encyclopedia of Entrepreneurship (pp. 72-90). Englewood Cliffs, N. J.: Prentice-Hall. 
Silva E. (1998). El rol del sistema universitario en la formación de los emprendedor. Managenment del emprendedor, Ed. Diario financiero.

Sommer, L. (2013). La influencia de la experiencia y el estilo cognitivo en las intenciones de emprendimiento internacional: la aportación de la educación académica en esta relación. Electronic Journal of Research in Educational Psychology, 11(2), 311-344. Doi: http://dx.doi.org/10.14204/ejrep.30.12167.

Tether, B.S. (2003). What is Innovation? Approaches to Distinguishing New Products and Services from Existing Products and Processes. ESRC Centre for Research on Innovation and Competition (CRIC), Working Paper 12. Manchester. Disponible en: http://www.cric.ac.uk/ cric/pdfs/wp12.pdf.

Thomas, A. S., \& Mueller, S. L., (2000). A case for comparative entrepreneurship: assessing the relevance of culture. Journal of International Business Studies, 31(2), 287-301. Disponible en: http://www.jstor.org/stable/155638.

Tolbert, R. S., David, R. J., \& Sine, W. D. (2011). Studying choice and change: the intersection of institutional theory and entrepreneurship research. Organization Science, 22 (5), 1332-1344. Doi: http://dx.doi.org/10.1287/orsc.1100.0601.

Turró A., Urbano, A., \& Peris-Ortiz, M. (2014). Culture and innovation: The moderating effect of cultural values on corporate entrepreneurship. Technological Forecasting \& Social Change, 88, 360-369. Doi: http://dx.doi.org/10.1016/j.techfore.2013.10.004.

Veciana, J. M., \& Urbano, D. (2004). Actitudes de los estudiantes universitarios hacia la creación de empresas: un estudio empírico comparativo entre Catalunya y Puerto Rico. El emprendedor innovador y la creación de empresas de I+D+I, Universidad de Valencia, 35-58.

Zahn-Waxler, C. (2000). The development of empathy, guilt, and internalization of distress. Implications for gender differences in internalizing and externalizing problems. En Davidson R, (eds.), Anxiety, Depression and Emotion: Wisconsin Symposium on Emotion, Vol. I. New York: Oxford Press, 222-265. 
Guzmán A. Muñoz Fernández et al.

[Página en blanco por razones de paginación]

- 66 - Electronic Journal of Research in Educational Psychology, 14(1), 45-66. ISSN: 1696-2095. 2016, no. 38 http://dx.doi.org/10.14204/ejrep.38.15040 